\title{
THE SNS FRONT-END, AN INJECTOR FOR A HIGH-POWER HYDROGEN-ION ACCELERATOR *
}

\author{
R. Keller \\ Ernest Orlando Lawrence Berkeley National Laboratory (LBNL), Berkeley, CA 94720, USA
}

\begin{abstract}
The Spallation Neutron Source** (SNS) will be an accelerator-based facility in Oak Ridge, TN, delivering pulsed neutron beams to experimenters. Negative hydrogen ion-beams are generated and pre-accelerated in a 2.5$\mathrm{MeV}$ linac injector, or front end (FE), accelerated to 1 $\mathrm{GeV}$ energy by a linear accelerator system, converted into protons and accumulated in a ring accelerator, and then directed towards a mercury target to generate the neutrons. The proton beam arrives at the target in bursts of less than $1 \mu$ s duration and with more than $1 \mathrm{MW}$ average power. The front end has been built and commissioned by LBNL in Berkeley; shipment to ORNL is essentially complete. This paper provides an overview of FE major design features and experimental results obtained during the commissioning process. The SNS-FE can be viewed as a prototype of a high-current, high duty-factor injector for other accelerator projects or, without the elaborate $\mathrm{MEBT}$, as an independent $2.5-\mathrm{MeV}$ accelerator for various applications.
\end{abstract}

\section{INTRODUCTION}

The SNS accelerator systems are comprehensively discussed elsewhere [1]. They aim at delivering intense proton-beam pulses of less than $1-\mu$ s duration to the spallation target at $60-\mathrm{Hz}$ repetition frequency and with an average beam power of $1.44 \mathrm{MW}$. The $1-\mathrm{ms}$ long $\mathrm{H}^{-}$macro pulses that are accelerated by the linac to $1-\mathrm{GeV}$ energy have to be chopped at about $1 \mathrm{MHz}$ frequency into 'mini pulses' of 645-ns duration, with 300-ns gaps.

LBNL has built the front end (linac injector) with its main beamline elements consisting of ion source, low-energy beam-transport section (LEBT), RFQ accelerator, and medium-energy beam-transport section (MEBT), as well as the ancillary systems needed to operate them. The beam- line, without ancillary systems, is shown in Fig. 1. Some parts of the SNS front end, i.e. the rf power system for the RFQ and the MEBT chopper structures and their power supplies, were supplied by LANL; some diagnostic elements and associated electronics by LANL and BNL, and personnel from these laboratories as well as from SNSORNL participated in the commissioning. The SNS Front-End project has been described in detail elsewhere with an ample collection of references [2, 3] and the present paper emphasizes the latest design features and commissioning results.

Beam chopping is performed by two separate chopper systems located in LEBT and MEBT, respectively. The LEBT chopper removes most of the beam power during the mini-pulse gaps, and the MEBT chopper reduces the rise and fall time of the transported beam to $10 \mathrm{~ns}$.

The main nominal parameters for the SNS Front End are listed in Table 1. The front end was assembled and commissioned at the Integrated Testing Facility at LBNL was shipped to ORNL in June/July, 2002.

\section{ION SOURCE AND LEBT}

The ion-source is shown in Fig. 2. The discharge plasma is sustained by pulsed 2-MHz-rf power and confined by a multi-cusp magnet configuration. Pulse ignition is facilitated by maintaining a stationary low-density plasma, driven by a separate $13.56-\mathrm{MHz}$ generator. A magnetic dipole filter separates the main plasma from a smaller $\mathrm{H}^{-}$production region where low-energy electrons help generating copious amounts of negative ions. A heated collar, equipped with eight cesium dispensers, surrounds this $\mathrm{H}^{-}$production volume, and a very thin (about $1 / 2$ mono layer) coating of cesium on the collar and outlet-electrode surfaces enhances the extracted beam current by a factor of three for a given rf power.

\footnotetext{
* Work supported by the Director, Office of Science, Basic Energy Sciences, US Department of Energy, Contract No. DE-AC03-76SF00098. ** SNS is a collaboration of six US Laboratories: Argonne National Laboratory (ANL), Brookhaven National Laboratory (BNL), Thomas Jefferson National Accelerator Facility (TJNAF), Los Alamos National Laboratory (LANL), E. O. Lawrence Berkeley National Laboratory (LBNL), and Oak Ridge National Laboratory (ORNL). SNS is managed by UT-Battelle, LLC, under contract DE-AC05-00OR22725 for U.S. Department of Energy. angle of about $3^{\circ}$ against the LEBT axis to compensate

Figure 1. The 9-m long SNS front-end beamline.

The outlet plate of the ion source contains a dipolemagnet configuration that creates a transverse deflecting field across the extraction gap, separates the extracted electrons from the ion beam and steers them towards a 'dumping' electrode biased at $5 \mathrm{kV}$ with respect to the outlet plate. Because this dumping field steers the ion beam as well, the entire plasma generator is tilted at an for this effect. The angle can be adjusted as needed.

Table 1. FES Nominal Performance Parameters

\begin{tabular}{lc}
\hline Ion species & $\mathrm{H}^{-}$ \\
Output energy (MeV) & 2.5 \\
$\begin{array}{l}\text { H- peak current: } \\
\text { MEBT output (mA) }\end{array}$ & 38 \\
$\quad \begin{array}{l}\text { Nominal ion-source output, } \\
\text { assuming 80\% RFQ transmission (mA) }\end{array}$ & 50 \\
Output normalized transverse rms emittance $(\pi \mathrm{mm} \mathrm{mrad})$ & 0.27 \\
Output normalized longitudinal rms emittance $(\pi \mathrm{MeV}$ deg) & 0.126 \\
Macro pulse length (ms) & 1 \\
Duty factor (\%) & 6
\end{tabular}
}




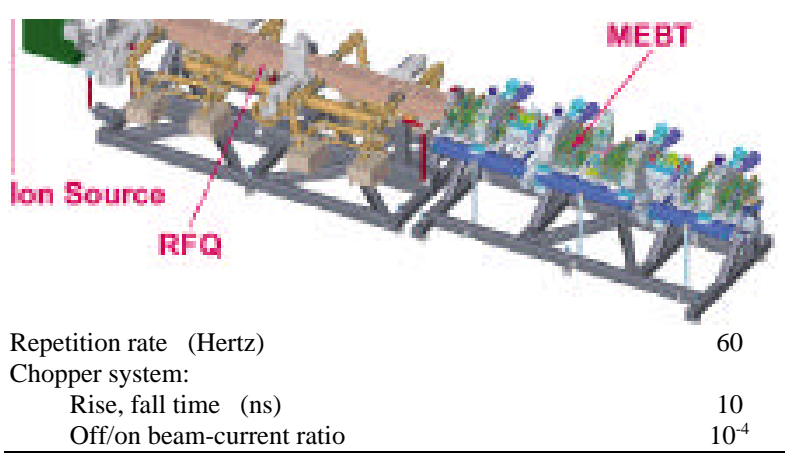

The LEBT serves the main purposes beam formation, 2-parameter matching into the RFQ, steering in angle and transverse offset, pre-chopping, and gas pumping. A fully electrostatic system with two einzel lenses as focusing elements was chosen for the SNS LEBT. The second one of these lenses is split into four quadrants that can be biased with d.c. and pulsed voltages to provide angular steering as well as pre-chopping. The LEBT can also be mechanically offset against the RFQ axis.

Average beam pulse-currents up to $50 \mathrm{~mA}$ have been transported through the LEBT at $6 \%$ duty factor during the dedicated commissioning phase for ion source and LEBT. Peak beam-current values up to $68 \mathrm{~mA}$ have been measured at the beginning of the pulses. The emittances show pronounced effects of aberration, but there is rather little beam current contained in the distorted wings.

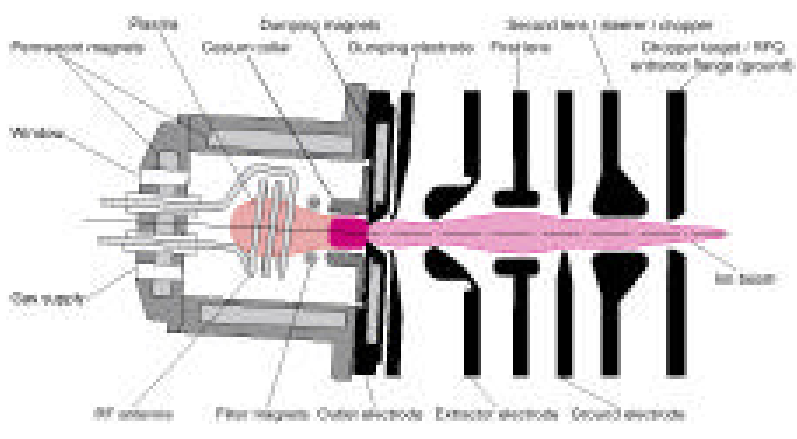

Figure 2. Ion source and LEBT.

\section{RFQ}

The 3.72-m long RFQ consists of four modules, built as composite structures with an outer GlidCop ${ }^{\circledR}$ shell enclosing four oxygen-free copper vanes. Peak surface fields reach $36 \mathrm{MV} / \mathrm{m}$, and the total $\mathrm{rf}$ power is $640 \mathrm{~kW}$ during pulses. Water-cooled $\pi$-mode stabilizers [4] separate unwanted dipole modes from the main quadrupole mode. Static frequency tuning is achieved by 20 slug tuners per module, and dynamic tuning by adjusting the temperature difference between vane tips and the outer walls of the modules.

All modules were conditioned together to full nominal rf gradient at $6 \%$ duty factor, and the measured field flatness is better than $\pm 1 \%$ peak-to-peak.

The RFQ was commissioned with beam at duty factors around $0.1 \%$. The main topics for this effort were transmission vs. rf power as shown in Fig. 3, transmission vs. injection energy, influence of LEBT steering and matching, and emittances vs. rf power and beam current.

Emittances were measured in one direction at a time, using a rotatable slit/wire-harp device. The effects of slit scattering as measured in dedicated tests have been subtracted from all RFQ (and later MEBT) emittance data, but no thresholding was applied on any of them. A measured vertical RFQ emittance is shown in Fig. 4.

A maximum beam current of $32 \mathrm{~mA}$ was recorded behind the RFQ with the ion-source extraction gap increased by $4 \mathrm{~mm}$ to obtain better LEBT matching at moderate currents. This result indicates an actual transmission through the RFQ above $90 \%$ and implicitly reduces the actual beam-current goal for the ion source to about $43 \mathrm{~mA}$, in order to reach 1.4-MW performance for the SNS accelerators.

The mini-pulse rise and fall times generated by the LEBT chopper were $25 \mathrm{~ns}$, twice as fast as had been assumed for the design of the MEBT chopper target.

Figure 3. Simulated and measured transmission values

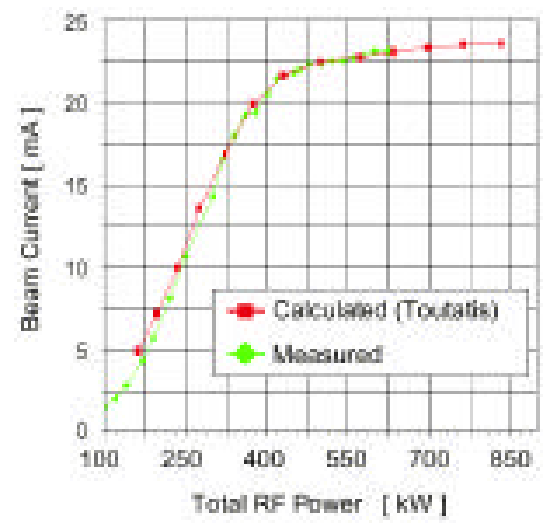

for the RFQ, with an input beam of 35-mA, show excellent agreement.

Figure 4. Vertical emittance of a 21.8-mA RFQ beam.

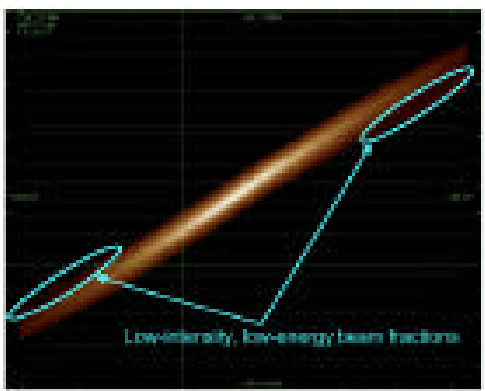

The measured rms size of $0.325 \pi \mathrm{mm}$ mrad includes a

$65-\mathrm{keV}$ beam fraction that carries very little current.

\section{MEBT}

The MEBT, shown in Figure 1, matches the beam from the RFQ through the MEBT chopper system and into the drift-tube linac currently being built by LANL. Fourteen quadrupole magnets and four rebuncher cavities provide transverse and longitudinal matching. An anti-chopper directs all particles back on axis that were deflected by 
the chopper during the rise and fall of the pulses and not fully intercepted by the target. Both chopper structures and their power supplies were provided by LANL.

Commissioning topics included beam transport and verification of the nominal 38-mA current without and with rebunchers; beam profiles; emittances; and the demonstration of $6 \%$ duty-factor operation. While the ion-source extraction gap was still $4 \mathrm{~mm}$ wider than nominal, $36 \mathrm{~mA}$ were initially transmitted. A round-theclock beam test was performed over one week to ascertain the reliability of all front-end subsystems in integrated operation. For the last few days of front-end commissioning, the ion-source gap was restored to its nominal width, and a maximum average pulse current of 50 $\mathrm{mA}$ was transported through the entire front end, exceeding the nominal current requirements by nearly $30 \%$.

MEBT emittances as the one seen in Fig. 5 show only slight signs of distortion, and the rms sizes are lower than those of corresponding RFQ emittances because the lowenergy particles are lost along the beamline.

Figure 5. Vertical emittance of a 19.5-mA beam at the
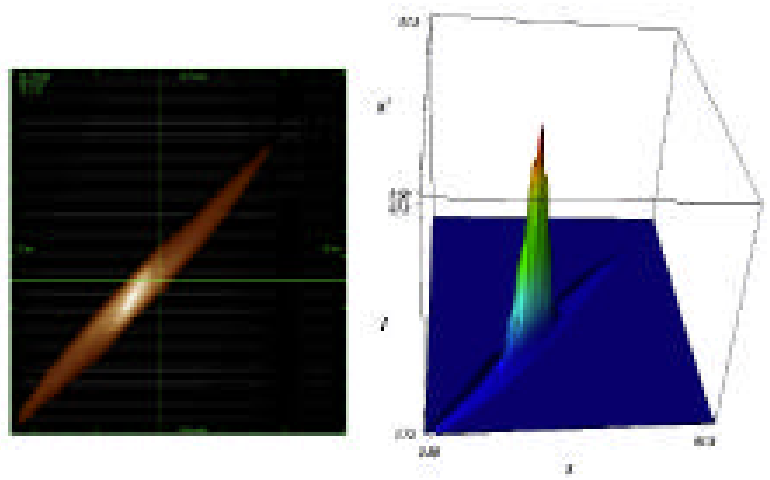

end of the MEBT. The rms size is $0.299 \pi \mathrm{mm}$ mrad.

\section{ACKNOWLEDGMENTS}

Main contributors to this work were J. J. Ayers, R. DiGennaro, L. Doolittle, R. Gough, J.B. Greer, S. Lewis, C. Lionberger, M. Monroy, J. Pruyn, A. Ratti, J.W. Staples, D. Syversrud, R. Thomae, and R. Yourd (LBNL); A. Aleksandrov, S. Assadi, P. Gibson, T. Shea, M. Stockli, R. Welton, and M. White (SNS-ORNL); J. Power and M. Stettler (LANL), and P. Cameron and C. Dawson (BNL). Thanks are due to all of them, as well as to many others whose names could not be listed in this space.

\section{REFERENCES}

[1] N. Holtkamp, "The SNS Linac and Storage Ring: Challenges and Progress Towards Meeting Them," Paper TUZGB002, EPAC 2002, Paris (2002).

[2] R. Keller for the FES Team, "Status of the SNS Front-End Systems, Paper MOP5B04, EPAC 2000, Wien (2000).

[3] R. Keller et al., "Progress with the SNS Front-End Systems," Paper MOPB005, PAC 2001, Chicago (2001).

[4] A. Ueno, et al., "Beam Test of the Pre-injector and the 3-MeV H RFQ with a New Field Stabilizer PISL," Linac '96, Geneva (1996). 